\title{
Effect of Acute Hypoxia on the Motor Activity and Heart Rate of the 10- and 14-Day Chick Embryo
}

\author{
Marina Nechaeva*, Irina Vladimirova and Tatyana Alexeeva
}

Laboratory of Developmental Biophysics, Institute of Developmental Biology RAS, Moscow, Russia

\begin{abstract}
Embryonic motility is an important component of development and may be a precursor of posthatching motor behavior. In chickens, it accompanies almost the entire embryogenesis and depends on environmental conditions, whose effects on embryonic motility have been poorly studied. The effect of acute hypoxia $\left(10 \% \mathrm{O}_{2}\right.$ for $\left.10 \mathrm{~min}\right)$ on the temporal parameters of chick embryo motility on incubation days 10 (D10) and 14 (D14) was estimated; the results were compared with data on the heart rate (HR). A force transducer connected with an embryo limb was used to record embryonic movements simultaneously with HR video recording. In the control, the duration of the activity phase (APh) and HR increased, while the inactivity phase (IPh) decreased in the period from D10 to D14. In response to hypoxia, the APh did not change significantly on either day, but the IPh significantly increased on D14 and tended to increase on D10. A distinct pattern of the motor activity response to hypoxia was observed on D14: the IPh increased after 0.5-2.5 min of hypoxia, peaked at a value 6 times as large as the control one, and then partly recovered. Under hypoxia, the mean HR significantly decreased to $87 \%$ of the control value and then partly recovered, increasing to $93 \%$ on both days studied. The similarity of the hypoxic patterns of IPh and HR on D14 suggests that the hypoxic recovery of HR contributes to the recovery of the embryo motility under hypoxia in late embryos.
\end{abstract}

Keywords: Chick embryo, motor activity, hypoxia, heart rate.

\section{INTRODUCTION}

Embryonic motor activity accompanies almost the entire embryogenesis in vertebrates and is an important component of ontogeny. It has been demonstrated to be necessary for the development of the musculoskeletal and nervous systems and disturbance of the embryonic movements leading to severe malformations and functional disorders [1-8]. It has also been supposed that embryonic motor activity is the precursor of motor behavior after birth or hatching $[9,10]$.

In the chicken, embryonic motor activity has been extensively studied [11-20]. It appears on incubation days 3-3.5, increases during development, and eventually transforms into targeted hatching behavior. During most of embryogenesis, this activity occurs in cycles consisting of the activity and inactivity phases (APh and $\mathrm{IPh}$, respectively). The spinal cord burst discharge has been found to be a good neural correlate of the general body motility.

It was shown earlier that embryonic motor activity depended on the environmental conditions, both chemical and physical, and it changed in response to fluctuations of temperature, illumination, acoustic stimulation, etc. $[5,21-$ 28]. However, the effects of environmental factors on the embryonic motor activity remain largely unknown. Oxygen is one of the most important environmental factors affecting development. Previous studies showed that a decreased oxygen level in the environment during embryogenesis (prenatal environmental hypoxia) led to considerable changes in both embryogenesis and postnatal life. It was

*Address correspondence to this author at the Institute of Developmental Biology RAS, Vavilov Str. 26, Moscow 119334, Russia; Tel: 7(499) 13563-27; Fax: 7(499) 135-80-12; E-mail: Mnechaeva2003@yahoo.com demonstrated that the developmental effect of hypoxia depended on the duration of exposure and degree of hypoxia, as well as the developmental stage. Nevertheless, the mechanism of the hypoxic effect on development remains unclear. Hence, we focused on the developmental effects of acute hypoxia in order to estimate how a short-term decrease in the ambient oxygen concentration affects embryonic motor activity at different developmental stages.

The effect of acute hypoxia during chick embryogenesis was extensively studied earlier. It was demonstrated that it induced a rapid, integrated response, numerous functional systems being involved in the prevention of damage to the embryo. This response includes a decrease in the $\mathrm{PO}_{2}$ of the blood; an increase in the blood catecholamine level; hypotensive bradycardia; redistribution of blood flow to vital organs, such as the brain, adrenals, and heart; and a decrease in the frequency of the amniotic contraction [29-35]. At the same time, changes in embryonic motility during acute hypoxia have been poorly studied. Therefore, our study on the changes in the embryonic motor activity during acute hypoxia may add to understanding its role in the integrated response of the embryo to hypoxia.

It is known that motor activity requires intense supply of energy and, hence, oxygen. The close interaction of the somatic movements and cardiovascular system in adults is generally known. In chick embryo, the cardiovascular system is involved in the transport of $\mathrm{O}_{2}$ from the gas exchange surface of the egg to embryonic tissues; its function substantially changes during acute hypoxia, depending on the developmental stage. We propose that hypoxic changes in the function of the cardiovascular system may contribute to the changes in the embryonic motility through alteration of the oxygen supply to the embryo. The 
heart rate (HR) is one of the most easily measured parameters of the cardiovascular system indirectly reflecting the changes in the embryonic blood flow. Hence, we decided to record simultaneously the changes in the HR and embryonic motiliy to estimate their relationship during acute hypoxia at different developmental stages.

Therefore, the goals of our study were to analyze the embryonic motor activity under normoxic conditions on incubation days 10 and 14 (D10 and D14, respectively) of chick embryogenesis, to estimate the changes in the motor activity in response to acute hypoxia at these stages, and to compare the changes in the embryonic motility during acute hypoxia with the changes in the embryonic HR.

\section{MATERIALS AND METHODS}

Fertile White Leghorn chicken eggs were obtained from a commercial supplier and incubated under normoxic conditions in a laboratory incubator at a temperature of $37.5 \pm$ $0.5^{\circ} \mathrm{C}$ and a humidity of $60-70 \%$.

Eggs were taken from the incubator on D10 or D14 and placed into a $300-\mathrm{ml}$ temperature-controlled $\left(37.5^{\circ} \mathrm{C}\right)$ humidified Plexiglas experimental chamber with a continuous flow of warm atmospheric air $(200 \mathrm{ml} / \mathrm{min})$. A force transducer was used to record embryonic movements in ovo [36]. Eggs were opened at the air-cell side by making a window approximately $2 \mathrm{~cm}$ in diameter in the egg shell above the developing embryo. A part of the outer shell membrane was removed, a small hole was cut in the chorioallantoic membrane and the amnion to reach the embryo, and the force transducer was attached to a limb of the embryo by means of a special grip (micro-serrefine). This method made it possible to accurately record the time course of the embryo motility. It allowed recording over a long time without damage to the embryo, noticeably limiting the embryo motility, or preventing the rotation out of the $x-y$ plane related to the amniotic rhythmic contractions.

The egg was allowed to stabilize for $30 \mathrm{~min}$. The embryonic movements were recorded sequentially in atmospheric air (control) for $30 \mathrm{~min}$, under hypoxia (in a mixture of $10 \% \mathrm{O}_{2}$ and $90 \% \mathrm{~N}_{2}$ supplied into the chamber at the same constant velocity of $200 \mathrm{ml} / \mathrm{min}$ ) for $10 \mathrm{~min}$, and again under normoxic conditions for $30 \mathrm{~min}$. We used a $10-$ min hypoxia because preliminary experiments with a 5-min exposure had not shown a distinct effect of acute hypoxia on embryo motility. A digital video camera mounted on a dissecting microscope was used to perform simultaneous video recording with a capture rate of $30 \mathrm{fps}$. It was used for additionally monitoring the embryonic movements and recording the HR. The mean durations of the $\mathrm{APh}$ and $\mathrm{IPh}$ were determined to quantitatively estimate the embryo motility. As in previous studies [19], we defined an APh as any episode of embryonic movements (no shorter than $6 \mathrm{~s}$ ) that was separated from other embryonic movements by at least $6 \mathrm{~s}$ of quiescence. We also studied the pattern of changes in the $\mathrm{APh}$ and $\mathrm{IPh}$ during the experiment and compared them with the changes in the HR. The HR was measured as a pulse rate in a major vessel with the use of time-lapse video recording.

All protocols of the manipulations with animals were approved by local or national bioethics committees. After the experiments, the embryos were staged according to the series for the chick embryo [37] and weighed.

All data were expressed as the mean \pm S.E.M. Statistical analysis was performed using Statistica (version 6.0, Statsoft). The $t$-test for independent samples was used to compare the mean values in different age groups (D10 and D14) and under different conditions (control and hypoxia). The nonparametric Wilcoxon matched-pairs test was used to estimate the significance of differences in HR under hypoxia. The differences were considered significant at $p<$ 0.05 .

\section{RESULTS}

\section{Embryonic Motor Activity and Heart Rate on D10 and D14 in Control}

To document the response to hypoxia we used twenty chick embryos at two different incubation times: D10 $(n=10)$ and D14 $(\mathrm{n}=10)$. The chick embryo reached the stage 36 on D10 and stage 40 on D14. Mean embryo mass increased from $2.50 \pm 0.21 \mathrm{~g}$ on D10 to $8.41 \pm 0.31 \mathrm{~g}$ on D14.

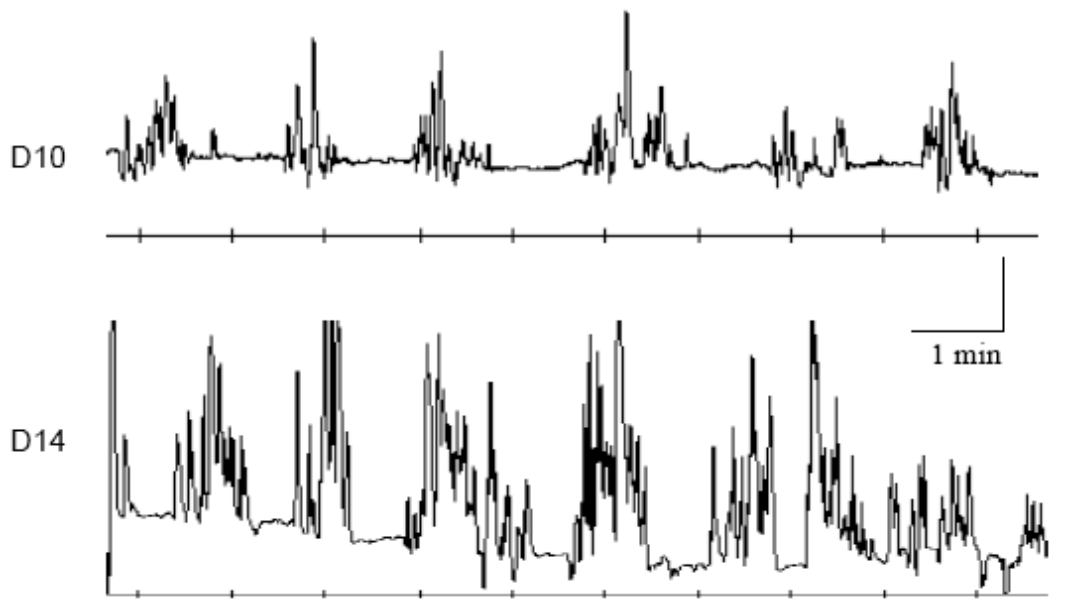

Fig. (1). Examples of the force recordings of the embryonic movements in ovo on incubation day 10 (D10) and day 14 (D14). Horizontal bar indicates time - 1min; vertical bar indicates amplitude $-0.25 \mathrm{mN}$ for D10 and $0.5 \mathrm{mN}$ for D14. 
The embryonic motility displayed cyclic character at these developmental stages and consisted of the $\mathrm{APh}$, which were separated by the IPh (Fig. 1). The APh plus the following $\mathrm{IPh}$ formed the cycle. During the $\mathrm{IPh}$, some rare simple movements of the parts of body and limbs or short periods of activity less than $6 \mathrm{~s}$ were observed, but they were not examined in this study. If the interval between the activities was less than $6 \mathrm{~s}$, it was added to the previous or following APh.

The duration of IPh significantly decreased from $41.8 \pm 4.9 \mathrm{~s}(\mathrm{n}=8)$ on $\mathrm{D} 10$ to $22.3 \pm 2.4 \mathrm{~s}(\mathrm{n}=10)$ on $\mathrm{D} 14$ $(\mathrm{F}=16.24 ; \mathrm{p}=0.001)$, while the APh significantly increased from $33.5 \pm 3.2 \mathrm{~s}(\mathrm{n}=8)$ on $\mathrm{D} 10$ to $44.7 \pm 2.0 \mathrm{~s}(\mathrm{n}=10)$ on D14 $(\mathrm{F}=9.79 ; \mathrm{p}=0.007)$ (Fig. 2A,B). The mean HR significantly increased from $236.0 \pm 5.9$ beat per min $(\mathrm{bpm})$ on $\mathrm{D} 10(\mathrm{n}=8)$ to $262.5 \pm 5.6 \mathrm{bpm}$ on $\mathrm{D} 14(\mathrm{n}=10)(\mathrm{F}=10.11 ; \mathrm{p}=0.006)$ (Fig. $3)$.

\section{Effect of Acute Hypoxia on Motor Activity and Heart Rate on D10 and D14}

The typical examples of the embryonic motor activity recordings during the experiment on D10 and D14 are presented on Fig. (4). Usually there were from 5 to 8 cycles during 10-minute hypoxia both on D10 and D14. Both on day 10 and D14, acute hypoxia did not change significantly the duration of the APh in comparison with the control value and during hypoxia the mean duration of $\mathrm{APh}$ was $32.9 \pm 2.3$ $\mathrm{s}(\mathrm{n}=8)$ on $\mathrm{D} 10(\mathrm{~F}=0.017 ; \mathrm{p}=0.89)$ and $39.3 \pm 2.2 \mathrm{~s}(\mathrm{n}=10)$ on $\mathrm{D} 14(\mathrm{~F}=3.153 ; \mathrm{p}=0.09)$ (Fig. 2A). At the same time, the mean duration of IPh significantly increased during hypoxia to $55.1 \pm 6.1 \mathrm{~s}(\mathrm{n}=10)$ on $\mathrm{D} 14(\mathrm{~F}=24.98 ; \mathrm{p}=0.00007)$ and tended to increase to $51.6 \pm 3.4 \mathrm{~s}$ on $\mathrm{D} 10 \quad(\mathrm{n}=8)(\mathrm{F}=2.616$; $\mathrm{p}=0.13$ ) (Fig. 2B). When the hypoxic mixture was replaced by air, both the APh and IPh did not differ significantly from the control level. Thus, the IPh was $48.1 \pm 4.2 \mathrm{~s}(\mathrm{n}=8)$ on D10 $(\mathrm{F}=0.943 ; \mathrm{p}=0.35)$ and $19.7 \pm 3.6 \mathrm{~s}(\mathrm{n}=10)$ on $\mathrm{D} 14(\mathrm{~F}=0.378$; $\mathrm{p}=0.55)$; the APh was $31.3 \pm 1.5 \mathrm{~s}(\mathrm{n}=8)$ on $\mathrm{D} 10(\mathrm{~F}=0.376$; $\mathrm{p}=0.55)$ and $45.1 \pm 1.7 \mathrm{~s}(\mathrm{n}=10)$ on $\mathrm{D} 14(\mathrm{~F}=0.022 ; \mathrm{p}=0.88)$. (Fig. 2A,B).
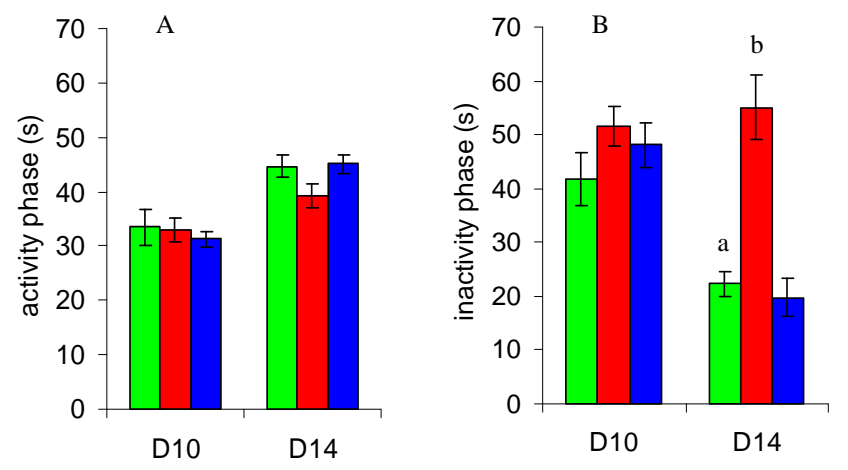

Fig. (2). Effect of acute hypoxia on the duration of the activity (A) and inactivity phases (B) of the chick embryo motor activity on incubation days 10 (D10) and day 14 (D14). Data are means \pm S.E.M. Green bars indicate values in normoxia (control), red bars - values under hypoxia and blue bars - recovery in air. Bars marked ' $a$ ' differ significanyly from those marked ' $b$ ' $(\mathrm{p}<0.05)$.

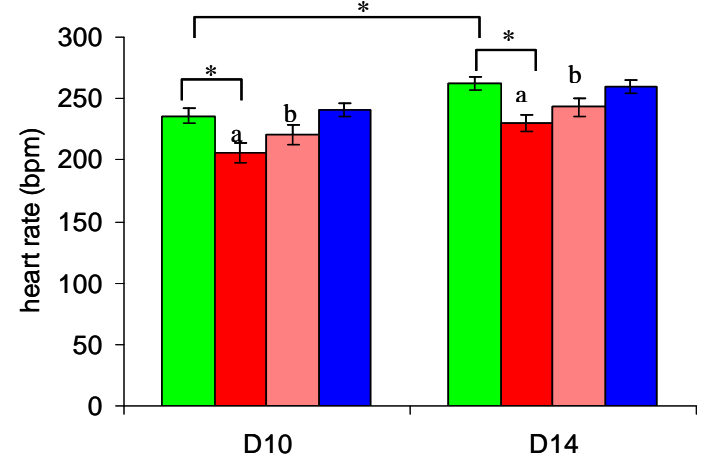

Fig. (3). Effect of acute hypoxia on the chick embryo heart rate on incubation days 10 (D10) and day 14 (D14). Data are means \pm S.E.M. Green bars indicate values in normoxia (control), red bars - minimum values under hypoxia; pink bars - partial recovery of heart rate under hypoxia and blue bars - recovery in the air. *Significant differences $(p<0.05)$. Bars marked 'a' differ significanyly from those marked ' $b$ ', Wilcoxon test.

To estimate the hypoxic response of the embryonic motility in more details, we analyzed the dynamics of $\mathrm{IPh}$ and $\mathrm{APh}$ during the hypoxic exposure; and such analysis showed the distinct differences in response for two ages studied. To describe the motor activity pattern under hypoxia the number of the cycle was presented on abscissa and the duration of $\mathrm{APh}$ or IPh on ordinate axis. To normalize these data 15 cycles before hypoxia, all cycles under hypoxia and then first 15 cycles during the recovery in the air were analyzed (Fig. 5).

A distinct pattern of the motor activity in response to hypoxia was observed on D14: the IPh increased after 0.5$2.5 \mathrm{~min}$ of hypoxia, peaked at a value $143.0 \pm 10.7 \mathrm{~s}(\mathrm{n}=10)$ (about 6 times as large as the control one), then partly recovered under hypoxic conditions and for the last two hypoxic cycles, the IPh was in average $38.0 \pm 5.9 \mathrm{~s}(\mathrm{n}=10)$, which was 1.7 times higher than in control $(\mathrm{F}=6.540$, $\mathrm{p}=0.02$ ). At the same time, there were no characteristic hypoxic patterns of the IPh on D10 and APh both on D10 and D14. We also observed individual variations of the hypoxic response on D10: in two embryos hypoxia produced the pattern similar to D14, but these embryos were not included for statistics.

Acute hypoxia caused the inhibitory effect on HR both on D10 and D14. Thus, the mean HR decreased by $12.8 \%$ on D10 and by $12.5 \%$ on D14 (Fig. 3). The dynamics of the HR changes during hypoxia was similar on both days studied. $\mathrm{HR}$ first decreased to 205.9 $\pm 7.9 \mathrm{bpm}(\mathrm{n}=8)$ on $\mathrm{D} 10$ $(\mathrm{F}=9.416, \mathrm{p}=0.009)$ and to $229.7 \pm 6.7 \mathrm{bpm}(\mathrm{n}=10)$ on $\mathrm{D} 14$ $(\mathrm{F}=14.012, \mathrm{p}=0.002)$. The time of the hypoxic response (which was defined as the time from the beginning of the hypoxic exposure to the moment when the HR reached its lowest value) was different significantly on D10 and D14; it averaged $146.4 \pm 17.1 \mathrm{~s} \quad(\mathrm{n}=8)$ and 94.5 $56.2 \mathrm{~s} \quad(\mathrm{n}=10)$, respectively $(\mathrm{F}=10.637, \mathrm{p}=0.005)$. Afterwards, the HR was partly restored against the background of continued hypoxia to $220.7 \pm 7.8 \mathrm{bpm}(\mathrm{n}=8)$ (Wilcoxon paired sample test, $\mathbf{p}=$ $0.028)$ on $\mathrm{D} 10$ and to $243.1 \pm 7.2 \mathrm{bpm}(\mathrm{n}=10)$ on $\mathrm{D} 14$ (Wilcoxon paired sample test, $\mathbf{p}=0.005$ ), which corresponded to 94 and $92 \%$ of the control HR values on days 10 and 14, respectively. After the hypoxic mixture was replaced with atmospheric air, the HR was completely restored and was $240.8 \pm 5.9 \mathrm{bpm}(\mathrm{n}=8)(\mathrm{F}=0.343 ; \mathrm{p}=0.34)$ on 


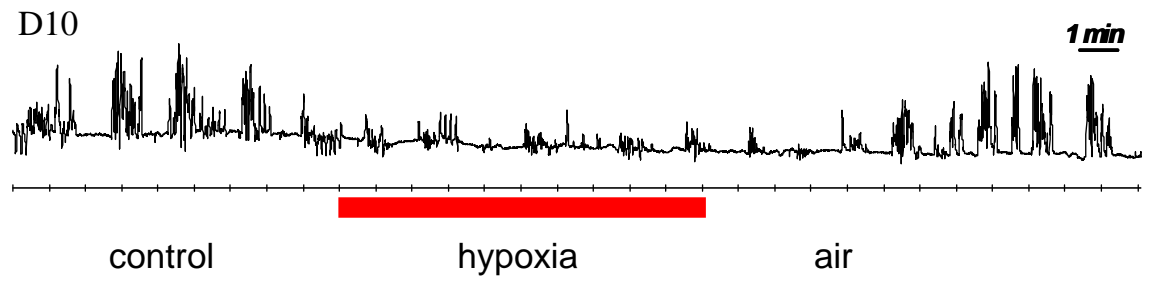

D14

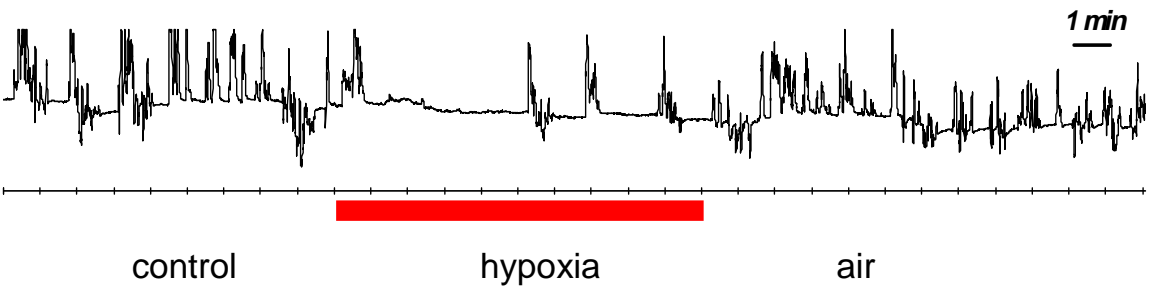

Fig. (4). Examples of the hypoxic patterns of the embryonic motility on incubation day 10 (D10) and day 14 (D14). Red line indicates the period of the hypoxic exposure during $10 \mathrm{~min}$. Bar $-1 \mathrm{~min}$.
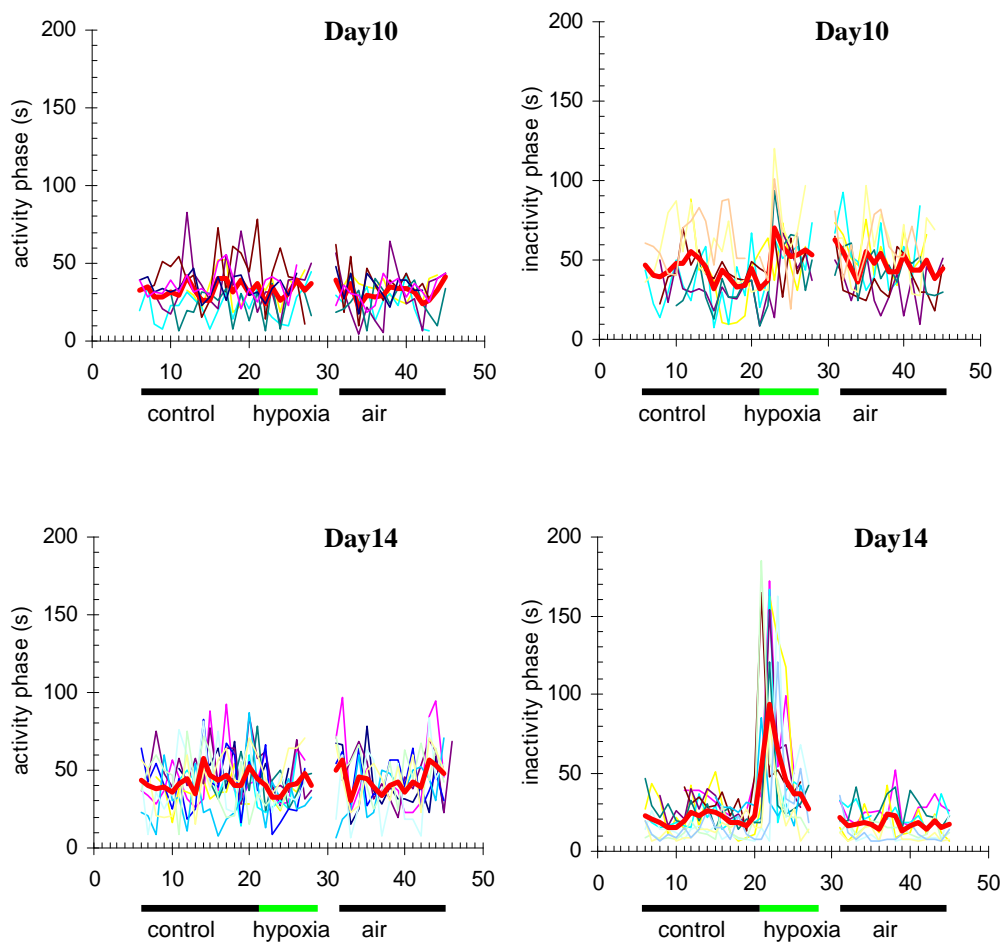

Fig. (5). Hypoxic patterns of the activity and inactivity phases of chick embryonic motility on incubation days 10 (D10) and day 14 (D14). Abscissa - number of cycle. To normalize data 15 cycles before hypoxia, then all cycles under hypoxia and first 15 cycles during the recovery in the air were ploted. Each trace - one experiment. The bold red line shows the mean values in 8 experiments on D10 and in 10 experiments on D14. Green bars indicate hypoxic exposure.

$\mathrm{D} 10$ and $259.3 \pm 5.3 \mathrm{bpm}(\mathrm{n}=10)$ on $\mathrm{D} 14(\mathrm{~F}=0.165 ; \mathrm{p}=0.69)$ (Fig. 3).

\section{DISCUSSION}

Embryonic motor activity is known to appear at early developmental stages of the chick embryo and accompany the entire subsequent embryogenesis. The role of embryonic motor activity remains largely unclear. Analysis of the pattern of changes in motor activity during embryogenesis and the effects of environmental factors on it may add to understanding its role in individual development.
Our study has shown that the chick embryo motor activity is cyclic both on D10 and D14, with periods of movement separated by periods of quiescence. This is confirmed by data on a cyclic pattern of embryonic motor activity earlier found by other researchers $[12,13,19,38$, 39]. Our data have also demonstrated that the embryo motility increases in the period from D10 to D14 as a result of both a decrease in the duration of the IPh and an increase in the duration of the APh. An increase in embryonic motor activity at about the same embryogenetic stage was also previously reported by other authors [13, 18, 19]. The absolute $\mathrm{IPh}$ and $\mathrm{APh}$ values determined in our study were close to the literature data for the corresponding period of 
development. We estimated the IPh duration on D10 at about $35 \mathrm{~s}$, which falls between 56 and $27 \mathrm{~s}$, the durations observed on D9 and D11, respectively; the IPh measured on D14 was comparable to that on D13 [19]. At the same time, the IPh on D9 estimated by Bradley [18] was almost three times as much as the value obtained in our experiments. Regarding $\mathrm{APh}$, our data for D10 are close to literature data for D9 [18, 19]; however, the $\mathrm{APh}$ on D14 was comparable to the literature data for D12 [18] but considerably shorter than those for D13 [19]. The differences in absolute values may have been related to different approaches to data processing, as well as different experimental procedures: other researchers recorded leg movements, whereas we studied the motor activity of the whole embryo. Thus, despite some differences in absolute values, the pattern of changes in the temporal parameters of the motor activity with embryo age observed in our study was similar to that reported by other authors.

It was earlier shown that many environmental factors, such as temperature, illumination, acoustic stimulation, vibration, etc., may considerably affect the motor activity of the chick embryo in the egg [21-28]. Since oxygen is one of the most important environmental factors affecting both physiological processes and development, our study was focused on the effect of oxygen deficit on embryonic motor activity. The motor activity of the embryo in the egg was demonstrated earlier to increase with increasing oxygen concentration and decrease with increasing $\mathrm{CO}_{2}$ concentration [40].

Our study showed that effect of acute hypoxia on the embryonic motor activity enhanced with age. The analysis of the time course of the response to acute hypoxia also showed a considerable difference between D10 and D14. Specifically, we observed a characteristic pattern of the response on D14: the motor activity first disappeared (i.e., the IPh duration increased, on average, by a factor of 6) and then was partly restored, so that the IP was only 1.7 times lower than in the control by the end the hypoxic exposure. On D10, two embryos exhibited hypoxic pattern typical for D14; but in most cases, only the mean IPh duration tended to increase. This leads us to the conclusion that the inhibitory effect of acute hypoxia on embryonic motor activity depend on the developmental stage, the embryo being able to partly resume the motor activity under hypoxic conditions on D14.

Since embryonic motor activity is neurogenic and the spinal cord burst discharge has been found to be a good neural correlate of the general body motility $[16,41]$, it is conceivable that the inhibitory effect of hypoxia on this motor activity is related to the effect of low oxygen on the activity of these neurons. The effect of acute environmental hypoxia with $10 \%$ of $\mathrm{O}_{2}$ on the spinal cord polyneuronal activity during the late stages of chick embryo incubation was earlier studied in ovo [42]. It has been demonstrated that a hypoxic environment inhibits the burst activity on D14. Thus, hypoxia may inhibit embryonic motor activity by suppressing neuron activity. Hypoxia may also cause changes at the levels of neuromuscular transmission and skeletal muscles, resulting in a decreased motor activity; however, this assumption has not been tested thus far.

The observed enhancement of the inhibitory effect of hypoxia on motor activity with age may be related to various factors and requires additional research. One should take into consideration that these developmental stages differ in the maturity of the motor system, especially that of the sensory systems incorporated into the spinal motor circuitry in this period [19]. In addition, the age-related changes may result from differences in the degree of development of the gas exchange and oxygen transport systems at the given stages of embryogenesis, with the result that similar levels of external hypoxia may lead to a decrease in the blood oxygen tension to different levels in embryos of different ages. For example, it has been shown that the gas exchange area is considerably increased as a result of chorioallantois growth [21] and blood circulation in the egg is accelerated [43] during this period of development. An increase in the embryo weight and oxygen consumption by the egg with age should also be taken into account. We found that the embryo weight increased by a factor of about three during the period from D10 to D14. Earlier, the oxygen consumption by the chicken egg was also found to increase by a factor of about three during the same period [44]. It may be assumed that the decrease in the air content of oxygen to $10 \%$ may have been critical to different degrees for 10- and 14-day-old embryos.

The restoration of the embryonic motor activity against a background of hypoxia that we observed in 14-day-old embryos is of special interest. Its mechanism remains unclear and requires further study. We may assume that this restoration was underlain by unidentified local mechanisms that, under the conditions of oxygen deficit, can improve the functions of the neurons responsible for embryonic motility by affecting, e.g., their metabolism (although these changes are usually slower). On the other hand, these may be some functional mechanisms in the egg that improve oxygen supply to nerve cells under hypoxia. We assumed that one of these functional mechanisms involves the cardiovascular system, which plays an important role in oxygen transport to embryo tissues. The effect may be mediated, e.g., by redistribution of blood circulation in the egg in such a way that oxygenated blood is preferentially distributed to the cerebral circulation, heart, and adrenal glands. However, this redistribution of circulation from the ancillary vascular beds toward myocardial, cerebral, and adrenal circulations in the chick embryo in response to hypoxia has been observed only at later stages of embryogenesis [30]. The increase in the blood flow rate may also have been resulted from an increase in the HR. We studied the time course of changes in the HR along with motor activity variation under hypoxic conditions. The pattern of changes in the HR was somewhat similar to that for motor activity on D14: after the hypoxiainduced decrease in the HR, it was partly restored against the background of hypoxic exposure, simultaneously with the restoration of motor activity. The partial restoration of the HR under hypoxia is likely to have increased the blood flow velocity and improved the oxygen supply to spinal neurons responsible for embryonic motor activity, which led to a partial restoration of their activity and allowed the embryo to resume motility. The pattern of the change in the HR on D10 was similar to that on D14; however, it was not similar to the course of the change in the motor activity. However, the D14-type changes in motor activity observed in two embryos on D10 followed about the same pattern as the changes in HR in them. 
The partial restoration of HR under hypoxia on D10 and D14 that followed its decrease may have been related to an increase in the catecholamine content of the blood under hypoxia resulting from stimulation of the adrenal glands [33]. However, we did not find published data on the effect of $10 \%$ hypoxia on the catecholamine content of the blood during the studied period of chick embryogenesis. However, it was shown that $100 \%$ hypoxia increased the NA concentration in the blood on D13 [31]. Similarly, a marked increase in the NA content of the blood in response to shortterm hypoxia $\left(13.5 \%\right.$ of $\left.\mathrm{O}_{2}\right)$ was detected on D11 [45].

More detailed analysis of our data on the changes in the HR in the course of chicken embryogenesis and under hypoxia has shown the following. The HR increases during the period from D10 to D14. Other authors earlier observed similar changes during about the same period of development [43, 46, 47]. In our study, the absolute HR was somewhat higher on D10 and somewhat lower on D14 [43, 47]. This may be explained by differences in the methods used for recording HR, as well as the temperatures at which the eggs were incubated and the experiment was performed. At both stages studied, hypoxia decreased the HR. This agrees with data obtained by other researchers [29]. The inhibitory effect on HR did not depend on the age; it was about the same on D10 and D14 (about $-30 \mathrm{bpm}$ ). These results agree with data on approximately the same period of development and a comparable level of hypoxia reported by other authors [29]. In those studies, however, no HR restoration during hypoxia was observed at the corresponding developmental stages. This may have been related to a shorter hypoxic exposure in those studies (5 min versus $10 \mathrm{~min}$ in our experiments), because the hypoxic restoration of the HR occurred usually on the fourth to sixth minute of our study. Comparison of the times of HR response to hypoxia on D10 and D14 showed that the HR more rapidly dropped under hypoxia on D14; i.e., the response time decreased with age. This may have been accounted for by a chemoreflex from aortal receptors and formation of parasympathetic innervations of the heart. Data on the stage at which the parasympathetic control of the HR is formed in the chick embryo are contradictory. Some authors believe that the parasympathetic control already exists as early as D14 [47, 48]. Others assume that parasympathetic control of the HR does not appear till end of embryogenesis [49]. The formation of tonic parasympathetic influence on the HR is incomplete at the ontogenetic stage dealt with in our study; however, it may already be expressed in a response to hypoxia on D14. Therefore, the inhibitory effect of hypoxia on HR on D10 is most likely to be determined by direct effect of low oxygen on the myocardium; on D14, the reflex component from aortal chemoreceptors may be added, which accelerates the onset of the inhibitory effect, leading to a more rapid decrease in the HR.

\section{CONCLUSION}

Thus, our data confirm the notion that the response to acute hypoxia is rapid and integrated, with many functional systems involved in it. It was earlier demonstrated to depend on the age of the chick embryo and it includes a complex changes in the different functional systems [29-35]. Our study has added data on the changes in embryonic motor activity to the general picture of the integrated response. Our results also could indicate that the response to acute hypoxia involves not only different functional systems affected by oxygen deficit, but also interactions between these systems, so that changes in one of them may alter the functions of others. For example, partial restoration of the HR under hypoxia may promote the resumption of embryonic motility. It is noteworthy that the response depends on the embryo age, which is accounted for by gradual development of both individual functional systems and regulatory mechanisms.

\section{ACKNOWLEDGEMENTS}

This research was partly supported by Grant RFBR 0804-01063a.

\section{REFERENCES}

[1] Hogg DA, Hosseini A. The effects of paralysis on skeletal development in the chick embryo. Comp Biochem Physiol 1992; 103A: 25-8.

[2] Bertram JE, Greenberg LS, Miyake T, Hall BK. Paralysis and long bone growth in the chick: growth shape trajectories of the pelvic limb. Growth Dev Aging 1997; 61: 51-60.

[3] Oppenheim RW, Prevette D, Houenou LJ, et al. Neuromuscular development in the avian paralytic mutant crooked neck dwarf $(\mathrm{cn} / \mathrm{cn})$ : further evidence for the role of neuromuscular activity in motoneuron survival. J Comp Neurol 1997; 381: 353-72.

[4] Bos AF, Einspieler C, Prechtl HF. Intrauterine growth retardation, general movements, and neurodevelopmental outcome: a review. Dev Med Child Neurol 2001; 43: 61-8.

[5] Müller GB. Embryonic motility: environmental influences and evolutionary innovation. Evol Dev 2003; 5: 56-60.

[6] Lamb KJ, Lewthwaite JC, Lin JP, et al. Diverse range of fixed positional deformities and bone growth restraint provoked by flaccid paralysis in embryonic chicks. Int J Exp Pathol 2003; 84: 191-9.

[7] Heywood JL, McEntee GM, Stickland NC. In ovo neuromuscular stimulation alters the skeletal muscle phenotype of the chick. J Muscle Res Cell Motil 2005; 26: 49-56.

[8] Pitsillides AA. Early effects of embryonic movement: 'a shot out of the dark'. J Anat 2006; 208: 417-31.

[9] Robinson SR. Behavioral embryology. In: Hopkins B, Ed. The cambridge encyclopedia of child development. Cambridge: Cambridge University Press 2005; pp. 469-73.

[10] Ryu YU, Bradley NS. Precocious locomotor behavior begins in the egg: development of leg muscle patterns for stepping in the chick. PLoS ONE 2009; 4(7): e6111.

[11] Hamburger V. Some aspects of the embryology of behavior. Q Rev Biol 1963; 38: 342-65.

[12] Hamburger V, Balaban M. Observation and experiments on spontaneous rhythmical behavior in the chick embryo. Dev Biol 1963; 7: 533-45.

[13] Hamburger V, Balaban M, Oppenheim R, Wenger E. Periodic motility of normal and spinal chick embryos between 8 and 17 days of incubation. J Exp Zool 1965; 159: 1-14.

[14] Oppenheim RW. Amniotic contraction and embryonic motility in the chick embryo. Science 1966; 152: 528-9.

[15] Provine RR. Ontogeny of bioelectric activity in the spinal cord of the chick embryo and its behavioral implications. Brain Res 1972; 41: 365-78.

[16] O'Donovan MJ. Motor activity in the isolated spinal cord of the chick embryo: Synaptic drive and firing pattern of single motoneurons. J Neurosci 1989; 9: 943-58.

[17] Bekoff A. Neuroethological approaches to the study of motor development in chicks: achievements and challenges. J Neurobiol 1992; 23: 1486-505.

[18] Bradley N. Transformations in embryonic motility in chick: kinematic correlates of type I and II motility at E9 and E12. J Neurophysiol 1999; 81: 1486-94.

[19] Sharp AA, Ma E, Bekoff A. Developmental changes in leg coordination of the chick at embryonic days 9, 11, and 13: 
uncoupling of ankle movements. J Neurophysiol 1999; 82: 240614.

[20] Bekoff A. Spontaneous embryonic motility: an enduring legacy. Int J Dev Neurosci 2001; 19: 155-60.

[21] Romanoff AL. The avian embryo. New York: Macmillan 1960.

[22] Wu KC, Streicher J, Lee ML, Hall BK, Müller GB. Role of motility in embryonic development. I. Embryo movements and amnion contractions in the chick and the influence of illumination. J Exp Zool 2001; 291: 186-94.

[23] Oppenheim RW, Levin HL. Short-term changes in incubation incubation temperature: behavioral and physiological effects in the chick embryo from 6 to 20 days. Dev Psychobiol 1975; 8: 103-15.

[24] Nechaeva MV, Turpaev TM. The effect of temperature on the motor activity of the chick embryo and amnion at 5-14 days of development. Zh Evol Biokhim Fiziol 1991; 27: 743-8.

[25] Bradley NS, Jahng DY. Selective effects of light exposure on distribution of motility in the chick embryo at E18. J Neurophysiol 2003; 90: 1408-17.

[26] Hammond CL, Simbi BH, Stickland NC. In ovo temperature manipulation influences embryonic motility and growth of limb tissues in the chick (Gallus gallus). J Exp Biol 2007; 210: 2667-75.

[27] Bursian AV. The influence of light on the spontaneous movements of chick embryos. Bull Exp Biol Med 1965; 58: 767-70.

[28] Sviderskaya GE. Effect of Sound and vibration on motor activity of chick embryos. Zh Evol Biokhim Fiziol 1968; 4: 251-7.

[29] van Golde J, Mulder T, Blanco CE. Changes in mean chorioallantoic blood flow and heart rate produced by hypoxia in the developing chick embryo. Pediatr Res 1997; 42: 293-8.

[30] Mulder ALM, van Golde JC, Prinzen FW, Blanco CE. Cardiac output distribution in response to hypoxia in the chick embryo in the second half of the incubation time. J Physiol 1998; 508: 281-7.

[31] Mulder ALM, van Golde JC, van Goor AAC, Giussani DA, Blanco CE. Developmental changes in plasma catecholamine concentrations during normoxia and acute hypoxia in the chick embryo. J Physiol 2000; 527: 593-9.

[32] Mulder ALM, van Goor CA, Giussani DA, Blanco CE. $\alpha$ Adrenergic contribution to the cardiovascular response to acute hypoxemia in the chick embryo. Am J Physiol Regul Integr Comp Physiol 2001; 281: 2004-10.

[33] Mulder ALM, Miedema A, De Mey JGR, Giussani DA, Blanco CE. Sympathetic control of the cardiovascular response to acute hypoxemia in the chick embryo. Am J Physiol Regul Integr Comp Physiol 2002; 282: 1156-63.

[34] Crossley II DA, Burggren WW, Altimiras J. Cardiovascular regulation during hypoxia in embryos of the domestic chicken Gallus gallus. Am J Physiol 2003; 284: 219-26.
[35] Nechaeva MV, Tönhardt H, Huhnke A, Makarenko IG, Tupraev TM. Effects of some environmental factors on the amnion rhythmic contractions in chick embryogenesis. Avian Poultry Biol Rev 2004; 15: $137-44$.

[36] Turpaev TM, Nechaeva MV, Solomonova VG. Motor activity of the chick embryo and amnion during embryogenesis. Zhurn Evol Brokhim Fiziol 1990; 26: 597- 605.

[37] Hamburger V, Hamilton HL. A series of normal stages in the development of the chick embryo. J Morphol 1951; 88: 49-92.

[38] Watson SJ, Bekoff A. A kinematic analysis of hindlimb motility in 9- and 10-day-old chick embryos. J Neurobiol 1990; 21: 651-60.

[39] Chambers SH, Bradley NS, Orosz MD. Kinematic analysis of wing and leg movements for type I motility in E9 chick embryos. Exp Brain Res 1995; 103: 218-26.

[40] Corner MA, Bot APC. Developmental patterns in the central nervous system of birds. Prog Brain Res 1967; 26: 214-36.

[41] O'Donovan M, Chub N. Population behavior and self-organization in the genesis of spontaneous rhythmic activity by developing spinal networks. Semin Cell Dev Biol 1997; 8: 21-8.

[42] Gonya-Magee T, Stokes BT. Acute modification of embryonic spinal cord activity induced by hypoxia. Dev Neurosci 1980; 3: 118.

[43] van Golde J, Mulder T, van Straaten H, Blanco CE. The chorioallantoic artery blood flow of the chick embryo from stage 34 to 43. Pediatr Res 1996; 40: 867-71.

[44] Romanoff AL. Biochemistry of the avian embryo. New York: Wiley 1967.

[45] Dragon S, Glombitza S, Götz R, Baumann R. Norepinephrinemediated hypoxic stimulation of embryonic red cell carbonic anhydrase and 2,3-DPG synthesis. Am J Physiol 1996; 271: 982-9.

[46] Tazawa H, Akiyama R, Pearson JT. Heart rate of developing avian embryos. In: Tönhardt H, Lewin R, Eds. The 3rd workshop: Investigation of perinatal development of birds: Berlin: Free University 1996; pp. 11-25.

[47] Höchel J, Akiyama R, Masuko T, Pearson JT, Nichelmann M, Tazawa H. Development of heart rate irregularities in chick embryos. Am J Physiol Heart 1998; 275: 527-33.

[48] Pappano AJ, Löffelholz K. Ontogenesis of adrenergic and cholinergic neuroeffector transmission in chick embryo heart. J Pharmacol Exp 1974; 191: 468-78.

[49] Crossley II DA, Altimiras J. Ontogeny of cholinergic and adrenergic cardiovascular regulation in the domestic chicken (Gallus gallus). Am J Physiol Regul Integr Comp Physiol 2000; 279: R1091-8.

(C) Nechaeva et al.; Licensee Bentham Open.

This is an open access article licensed under the terms of the Creative Commons Attribution Non-Commercial License (http://creativecommons.org/licenses/by$\mathrm{nc} / 3.0 /$ ), which permits unrestricted, non-commercial use, distribution and reproduction in any medium, provided the work is properly cited. 